\title{
Consensus of Continuous-Time Multiagent Systems with General Linear Dynamics and Nonuniform Sampling
}

\author{
Yanping Gao, ${ }^{1}$ Bo Liu, ${ }^{2}$ Min Zuo, ${ }^{1}$ Tongqiang Jiang, ${ }^{1}$ and Junyan $\mathrm{Yu}^{3}$ \\ ${ }^{1}$ Beijing Technology and Business University, Beijing 100048, China \\ ${ }^{2}$ North China University of Technology, Beijing 100144, China \\ ${ }^{3}$ University of Electronic Science and Technology of China, Chengdu 610054, China
}

Correspondence should be addressed to Bo Liu; boliu@ncut.edu.cn

Received 29 March 2013; Accepted 16 May 2013

Academic Editor: Wenwu Yu

Copyright (C) 2013 Yanping Gao et al. This is an open access article distributed under the Creative Commons Attribution License, which permits unrestricted use, distribution, and reproduction in any medium, provided the original work is properly cited.

\begin{abstract}
This paper studies the consensus problem of multiple agents with general linear continuous-time dynamics. It is assumed that the information transmission among agents is intermittent; namely, each agent can only obtain the information of other agents at some discrete times, where the discrete time intervals may not be equal. Some sufficient conditions for consensus in the cases of state feedback and static output feedback are established, and it is shown that if the controller gain and the upper bound of discrete time intervals satisfy certain linear matrix inequality, then consensus can be reached. Simulations are performed to validate the theoretical results.
\end{abstract}

\section{Introduction}

Consensus is one of the fundamental issues in the study of distributed control of multiagent systems, and it has wide applications in formation control of multiple robots, communication among sensor networks, cooperative control of unmanned aerial vehicles, and so forth. Much research work on consensus has been emerged, and most of the existing work focuses on the consensus problems of multiple agents with special dynamics, such as first-order dynamics (or single integrator) [1-10], second-order dynamics (or double integrators) [11-19], and high-order dynamics [20, 21]. Consensus problems of second-order continuous-time multiagent systems are studied in [12-14], where the information transmission among agents is intermittent. In [15-17], only the partial state of second-order agent can be measured, and thus, some static or dynamic output feedback controllers are designed. In [20-22], consensus is studied for high-order and nonlinear multiagent systems, respectively.

In recent years, the consensus of multiple agents with general linear dynamics has been paid more and more attention, such as [23-28], and the analysis of such multiagent systems is more challenging than the case of special dynamics. In [24], consensus is considered in the case of static output feedback, and it is proved that the consensus is equivalent to the Hurwitz stable or Schur stable of a constant matrix, which is determined by the topology and the system dynamics. By studying the stability of the constant matrix, it is shown that consensus can be reached for continuous-time multiagent systems if and only if the system is stabilizable and detectable and the topology has a spanning tree under some rank constraints, and a necessary condition is also provided for consensus of discrete-time multiagent systems. In [25], consensus of continuous-time and discrete-time multiagent systems under a dynamic output feedback controller, which is actually a state estimator, is investigated, respectively. By applying the result in [29], some sufficient and necessary conditions are presented in [25]. In [26], the joint effect of network topology, agent dynamics, and communication data rate on consensus of discrete-time multiagent systems is analyzed, and it is shown that under perfect state feedback, consensus is reached if and only if the dynamics of each agent is stabilizable and the unstable eigenvalues of each agent satisfy some constraints. In [27], the $H_{\infty}$ consensus problem of continuous-time multiagent systems is studied under dynamic output feedback by applying the robust 
control theory and linear matrix inequality technique. In [28], consensus of discrete-time multiagent systems under a dynamic output feedback controller, which is actually an observer-type controller, is discussed, and the discrete-time consensus region is analyzed for neurally stable agents and unstable agents, respectively.

It should be mentioned that $[23-25,27]$ all study the consensus of continuous-time multiagent systems, and the information transmission among agents is continuous. However, due to the limitation of bandwidth, the cost of communication, the technique constraints, and so forth, it is possible to transmit information in the intermittent manner. In addition, sampled-data control has many favorable properties, such as flexibility, robustness, and low cost see [30] for further details. Hence, it is also necessary to study the consensus of general continuous-time multiagent systems with intermittent information transmission. To the authors' best knowledge, there is little research work reported on this problem. Based on the previous consideration, we analyze the consensus of continuous-time multiagent systems with general dynamics, where each agent can only obtain the information of other agents at discrete times. Moreover, the discrete time intervals may not be equal, which often occurs in the event-driven systems or networked control systems [31]. The sufficient condition for consensus and the method to design controller gain are presented.

This paper is organized as follows. In Section 2, we present some concepts in graph theory and formulate the model to be studied. In Section 3, main results are stated. In Section 4, simulations are provided to illustrate the effectiveness of the theoretical results. Conclusion remarks are made in Section 5.

Notations. Let $I$ or $I_{n} \in \mathbb{R}^{n \times n}$ be an identity matrix, and $\mathbf{1}_{n}=[1, \ldots, 1]^{T} \in \mathbb{R}^{n}$; for symmetric matrices $A$ and $B$, $A<B$ (resp., $A \leq B$ ) means that $A-B$ is a negative definite (resp., negative semidefinite) matrix; $\otimes$ denotes the Kronecker product operator, and $C \otimes D=\left[c_{i j} D\right]$, where $C=\left[c_{i j}\right]$ and $D=\left[d_{i j}\right]$ are two matrices.

\section{Preliminaries}

2.1. Graph Theory. Some basic definitions in graph theory [32] are first introduced.

A directed graph $\mathscr{G}$ consists of a vertex set $\mathscr{V}(\mathscr{G})$ and an edge set $\mathscr{E}(\mathscr{G})$, where $\mathscr{V}(\mathscr{G})=\left\{v_{1}, \ldots, v_{n}\right\}$ and $\mathscr{E}(\mathscr{G}) \subset$ $\left\{\left(v_{j}, v_{i}\right): v_{j}, v_{i} \in \mathscr{V}(\mathscr{G})\right\}$. For edge $\left(v_{j}, v_{i}\right), v_{j}$ is called the parent vertex of $v_{i}$ and $v_{i}$ is called the child vertex of $v_{j}$. The set of neighbors of vertex $v_{i}$ is defined by $N\left(\mathscr{G}, v_{i}\right)=\left\{v_{j}\right.$ : $\left(v_{j}, v_{i}\right) \in \mathscr{E}(\mathscr{G})$ and $\left.j \neq i\right\}$, and the associated index set is denoted by $N(\mathscr{G}, i)=\left\{j: v_{j} \in N\left(\mathscr{G}, v_{i}\right)\right\}$. A (directed) path from $v_{i_{1}}$ to $v_{i_{k}}$ is a sequence, $v_{i_{1}}, \ldots, v_{i_{k}}$, of distinct vertices such that $\left(v_{i_{j}}, v_{i_{j+1}}\right) \in \mathscr{E}(\mathscr{G}), j=1, \ldots, k-1$. A directed graph $\mathscr{G}$ is strongly connected if there is a path from every vertex to every other vertex. A directed tree is a directed graph, where every vertex except one special vertex has exactly one parent vertex, and the special vertex, called root vertex, has no parent vertices and can be connected to any other vertices via paths. A subgraph $\mathscr{G}_{s}$ of $\mathscr{G}$ is a graph such that $\mathscr{V}\left(\mathscr{G}_{s}\right) \subset \mathscr{V}(\mathscr{G})$ and $\mathscr{E}\left(\mathscr{G}_{s}\right) \subset \mathscr{E}(\mathscr{G}) . \mathscr{G}_{s}$ is said to be a spanning subgraph if $\mathscr{V}\left(\mathscr{G}_{s}\right)=\mathscr{V}(\mathscr{G})$. For any $v_{i}, v_{j} \in \mathscr{V}\left(\mathscr{G}_{s}\right)$, if $\left(v_{i}, v_{j}\right) \in \mathscr{E}\left(\mathscr{G}_{s}\right) \Leftrightarrow$ $\left(v_{i}, v_{j}\right) \in \mathscr{E}(\mathscr{G})$, then $\mathscr{G}_{s}$ is said to be an induced subgraph of $\mathscr{G}$ and $\mathscr{G}_{s}$ is also said to be induced by $\mathscr{V}\left(\mathscr{G}_{s}\right)$. A spanning tree of $\mathscr{G}$ is a directed tree which is a spanning subgraph of $\mathscr{G} \mathscr{G}$ is said to have a spanning tree if some edges form a spanning tree of $\mathscr{G}$.

A matrix is called nonnegative if each of its elements is nonnegative. A weighted directed graph $\mathscr{G}(F)$ is a directed graph $\mathscr{G}$ plus a nonnegative matrix $F=\left[f_{i j}\right] \in \mathbb{R}^{n \times n}$, where $f_{i j}>0 \Leftrightarrow\left(v_{j}, v_{i}\right) \in \mathscr{E}(\mathscr{G})$, and $f_{i j}$ is called the weight of edge $\left(v_{j}, v_{i}\right)$. The Laplacian matrix $L=\left[l_{i j}\right] \in \mathbb{R}^{n \times n}$ of $\mathscr{G}(f)$ is defined as

$$
l_{i j}= \begin{cases}-f_{i j}, & i \neq j, \\ \sum_{s=1, s \neq i}^{n} f_{i s}, & i=j .\end{cases}
$$

The Laplacian matrix of $\mathscr{G}(F)$ has the following properties.

\section{Lemma 1 (see [2]).}

(i) Zero is an eigenvalue of $L$, and $\mathbf{1}_{n}$ is the associated right eigenvector.

(ii) Zero is an algebraically simple eigenvalue of $L$, and all the other eigenvalues are with positive real parts if and only if $\mathscr{G}(F)$ has a spanning tree.

2.2. Model. Consider a group of agents with the following general continuous-time dynamics:

$$
\begin{gathered}
\dot{x}_{i}(t)=A x_{i}(t)+B u_{i}(t), \\
y_{i}(t)=C x_{i}(t), \quad i=1,2, \ldots, n,
\end{gathered}
$$

where $x_{i} \in \mathbb{R}^{m}, u_{i} \in \mathbb{R}^{p}$ and $y_{i} \in \mathbb{R}^{q}$, are the state, control input, and output of agent $i$, respectively, and $A, B, C$ are constant matrices.

Given $u_{i}, i=1, \ldots, n$, we say that $u_{i}$ or multiagent system (2) solves a consensus problem asymptotically if $\lim _{t \rightarrow \infty}\left(x_{i}(t)-x_{j}(t)\right)=0, i, j=1,2, \ldots, n$, for any initial states.

In this paper, we consider the case of intermittent information transmission; namely, each agent can only obtain the information of its neighbors at some discrete times $t_{0}, t_{1}, \ldots$, where $0 \leq t_{0}<t_{1}<t_{2}<\cdots$. Let $T_{k}=t_{k+1}-t_{k}$, and assume that $T_{k} \leq d, k=0,1, \ldots$; namely, all discrete time intervals have a common upper bound. Note that all $T_{k}$ may not be equal.

In the case that the (relative) state of each agent can be measured directly, we consider the following control input:

$$
\begin{array}{r}
u_{i}(t)=K \sum_{j \in N(\mathscr{G}, i)} a_{i j}\left(x_{j}\left(t_{k}\right)-x_{i}\left(t_{k}\right)\right), \\
t \in\left[t_{k}, t_{k+1}\right), i=1,2, \ldots, n,
\end{array}
$$

where $K$ is the controller gain to be designed. 
In some times, the state of each agent cannot be measured directly, and thus, we also consider the following static output feedback controller:

$$
\begin{array}{r}
u_{i}(t)=K \sum_{j \in N(\mathscr{G}, i)} a_{i j}\left(y_{j}\left(t_{k}\right)-y_{i}\left(t_{k}\right)\right), \\
t \in\left[t_{k}, t_{k+1}\right), i=1,2, \ldots, n .
\end{array}
$$

Remark 2. Obviously, if $A$ is Hurwitz stable, then consensus can be reached for $K=0$. Hence, we assume that $A$ is not Hurwitz stable in this paper.

\section{Main Results}

In this section, we will present a sufficient condition for consensus under controllers (3) and (4), respectively, and the methods to design controller gains are also provided.

Let $X(t)=\left(x_{1}^{T}(t), x_{2}^{T}(t), \ldots, x_{n}^{T}(t)\right)^{T}$, then multiagent system (2) under controller (3) can be written as

$$
\begin{array}{r}
\dot{X}(t)=\left(I_{n} \otimes A\right) X(t)-(L \otimes(B K)) X\left(t_{k}\right), \\
t \in\left[t_{k}, t_{k+1}\right), \quad k=0,1, \ldots
\end{array}
$$

By Lemma 1, there exists an invertible matrix $U$, the first column of which is $\mathbf{1}_{n}$, such that

$$
U^{-1} L U=\left(\begin{array}{cc}
0 & \alpha \\
0 & H
\end{array}\right)
$$

where $H \in \mathbb{R}^{(n-1) \times(n-1)}$. Let $\delta(t)=\left(U^{-1} \otimes I_{m}\right) X(t)$; then

$$
\begin{gathered}
\dot{\delta}_{1}(t)=A \delta_{1}(t)-(\alpha \otimes(B K)) \tilde{\delta}\left(t_{k}\right), \\
\dot{\tilde{\delta}}(t)=\left(I_{n-1} \otimes A\right) \widetilde{\delta}(t)-(H \otimes(B K)) \widetilde{\delta}\left(t_{k}\right),
\end{gathered}
$$

where $\delta(t)=\left(\delta_{1}(t)^{T}, \widetilde{\delta}(t)^{T}\right)^{T}, \delta_{1}(t) \in \mathscr{R}^{m}$.

By the previous state transformation, it is easy to obtain the following lemma.

Lemma 3. Controller (3) solves a consensus problem asymptotically if and only if system (8) is asymptotically stable.

Proof. Sufficiency. Let $U=\left[\mathbf{1}_{n}, U_{1}\right]$, where $U_{1} \in \mathscr{R}^{n \times(n-1)}$. By $X(t)=\left(U \otimes I_{m}\right) \delta(t)$, we have $X(t)=\mathbf{1}_{n} \otimes \delta_{1}(t)+$ $\left(U_{1} \otimes I_{m}\right) \tilde{\delta}(t)$. Clearly, if system (8) is asymptotically stable, namely, $\lim _{t \rightarrow \infty} \widetilde{\delta}(t)=0$, then $\lim _{t \rightarrow \infty}\left(x_{i}(t)-x_{j}(t)\right)=0$, $i, j=1,2, \ldots, n$.

Necessity. Let $U^{-1}=\left(u_{2}, U_{2}^{T}\right)^{T}$, where $U_{2} \in \mathscr{R}^{(n-1) \times n}$. From $U^{-1} U=I_{n}$, we have $u_{2}^{T} \mathbf{1}_{n}=1, U_{2} \mathbf{1}_{n}=0$. Since consensus is reached, there exists $\phi(t) \in \mathscr{R}^{m}$ such that $\lim _{t \rightarrow \infty}\left(x_{i}(t)-\right.$ $\phi(t))=0, i=1,2, \ldots, n$. By $\delta(t)=\left(U^{-1} \otimes I_{m}\right) X(t)$,

$$
\begin{aligned}
\delta(t)- & \left(U^{-1} \otimes I_{m}\right)\left(\mathbf{1}_{n} \otimes \phi(t)\right) \\
& =\left(U^{-1} \otimes I_{m}\right)\left(X(t)-\mathbf{1}_{n} \otimes \phi(t)\right) \\
& \Longrightarrow \delta(t)-\left(\begin{array}{c}
u_{2}^{T} \mathbf{1}_{n} \\
U_{2} \mathbf{1}_{n}
\end{array}\right) \otimes \phi(t) \\
& =\left(U^{-1} \otimes I_{m}\right)\left(X(t)-\mathbf{1}_{n} \otimes \phi(t)\right) \\
& \Longrightarrow \delta(t)-\left(\begin{array}{l}
1 \\
0
\end{array}\right) \otimes \phi(t) \\
& =\left(U^{-1} \otimes I_{m}\right)\left(X(t)-\mathbf{1}_{n} \otimes \phi(t)\right) \\
& \Longrightarrow\left(\begin{array}{c}
\delta_{1}(t)-\phi(t) \\
\tilde{\delta}(t)
\end{array}\right) \\
& =\left(U^{-1} \otimes I_{m}\right)\left(X(t)-\mathbf{1}_{n} \otimes \phi(t)\right) \\
& \Longrightarrow \lim _{t \rightarrow \infty}\left(\begin{array}{c}
\delta_{1}(t)-\phi(t) \\
\tilde{\delta}(t)
\end{array}\right) \\
= & \left(\begin{array}{l}
u_{2}^{T} \otimes I_{m} \\
U_{2} \otimes I_{m}
\end{array}\right) \lim _{t \rightarrow \infty}\left(X(t)-\mathbf{1}_{n} \otimes \phi(t)\right) .
\end{aligned}
$$

By $\lim _{t \rightarrow \infty}\left(X(t)-\mathbf{1}_{n} \otimes \phi(t)\right)=0$, it is easy to obtain $\lim _{t \rightarrow \infty} \widetilde{\delta}(t)=0$, which means that system (8) is asymptotically stable.

By Lemma 3, we will analyze the stability of system (8) by applying the input delay approach [33], which is an effective method to deal with the stability of continuous-time systems with intermittent input.

Let $\tau(t)=t-t_{k}$, and let $t \in\left[t_{k}, t_{k+1}\right)$; then $t_{k}=t-\tau(t)$, and system (8) can be rewritten as

$$
\begin{aligned}
\dot{\widetilde{\delta}}(t)= & \left(I_{n-1} \otimes A\right) \widetilde{\delta}(t)-(H \otimes(B K)) \widetilde{\delta}(t-\tau(t)) \\
= & \left(I_{n-1} \otimes A-H \otimes(B K)\right) \widetilde{\delta}(t) \\
& +(H \otimes(B K))(\widetilde{\delta}(t)-\widetilde{\delta}(t-\tau(t))) \\
= & \widetilde{A} \tilde{\delta}(t)+(H \otimes(B K)) \xi(t),
\end{aligned}
$$

where $\widetilde{A}=I_{n-1} \otimes A-H \otimes(B K), \xi(t)=\widetilde{\delta}(t)-\widetilde{\delta}(t-\tau(t))$.

By $T_{k} \leq d$, we have $0 \leq \tau(t) \leq d, k=0,1, \ldots$. Obviously, the stability of system (8) is equivalent to that of system (10). By analyzing the stability of system (10), we obtain the following main result.

Theorem 4. Assume that $(A, B)$ is stabilizable and the topology graph has a spanning tree. Controller (3) solves a consensus problem asymptotically if there exist positive definite matrices 
$P, R$ such that $K$ and $d$ satisfy the following linear matrix inequality:

$$
\left(\begin{array}{ccc}
P \widetilde{A}+\widetilde{A}^{T} P & P(H \otimes(B K)) & \widetilde{A}^{T} R \\
* & -\frac{1}{d} R & (H \otimes(B K))^{T} R \\
* & * & -\frac{1}{d} R
\end{array}\right) .
$$

Proof. Consider the following Lyapunov-Krasovskii functional for system (10):

$$
V(t)=\widetilde{\delta}^{T}(t) P \widetilde{\delta}(t)+\int_{t-d}^{t}(s-t+d) \dot{\tilde{\delta}}^{T}(s) R \dot{\tilde{\delta}}(s) d s,
$$

where $P>0, R>0$, and then

$$
\begin{aligned}
\dot{V}(t)= & 2 \widetilde{\delta}^{T}(t) P \dot{\widetilde{\delta}}(t) \\
& +\left(-\int_{t-d}^{t} \dot{\widetilde{\delta}}^{T}(s) R \dot{\widetilde{\delta}}(s) d s+d \dot{\widetilde{\delta}}^{T}(t) R \dot{\widetilde{\delta}}(t)\right) .
\end{aligned}
$$

By Lemma 4 in [34],

$$
\begin{aligned}
& \int_{t-d}^{t} \dot{\tilde{\delta}}^{T}(s) R \dot{\tilde{\delta}}(s) d s \\
& \quad \geq \frac{1}{d}(\widetilde{\delta}(t)-\widetilde{\delta}(t-\tau(t)))^{T} R(\widetilde{\delta}(t)-\widetilde{\delta}(t-\tau(t))),
\end{aligned}
$$

and thus,

$$
\begin{aligned}
\dot{V}(t) \leq & 2 \widetilde{\delta}^{T}(t) P \dot{\tilde{\delta}}(t) \\
& -\frac{1}{d}(\widetilde{\delta}(t)-\widetilde{\delta}(t-\tau(t)))^{T} R(\widetilde{\delta}(t)-\widetilde{\delta}(t-\tau(t))) \\
& +d \dot{\widetilde{\delta}}^{T}(t) R \dot{\widetilde{\delta}}(t) \\
= & \left(\widetilde{\delta}^{T}(t), \xi^{T}(t)\right) Q\left(\widetilde{\delta}^{T}(t), \xi^{T}(t)\right)^{T},
\end{aligned}
$$

where

$$
\begin{aligned}
Q= & \left(\begin{array}{cc}
P \widetilde{A}+\widetilde{A}^{T} P & P(H \otimes(B K)) \\
* & -\frac{1}{d} R
\end{array}\right) \\
& +d\left(\begin{array}{c}
\widetilde{A}^{T} \\
(H \otimes(B K))^{T}
\end{array}\right) R(\widetilde{A} H \otimes(B K)) .
\end{aligned}
$$

By Schur complement, $Q<0$ if and only if (11) is satisfied. Hence, $\dot{V}(t)<0$; namely, system (10) is asymptotically stable. By Lemma 3, consensus is reached.

Remark 5. By [24], if $(A, B)$ is stabilizable and the topology graph has a spanning tree, then there exists $K$ such that $\widetilde{A}$ is Hurwitz stable; namely, there exists $P>0$ such that $P \widetilde{A}+$ $\widetilde{A}^{T} P<0$. Obviously, if $d>0$ is small enough, then (11) must be satisfied. Hence, by (11), we can find $K$ and $d$ which ensure consensus.

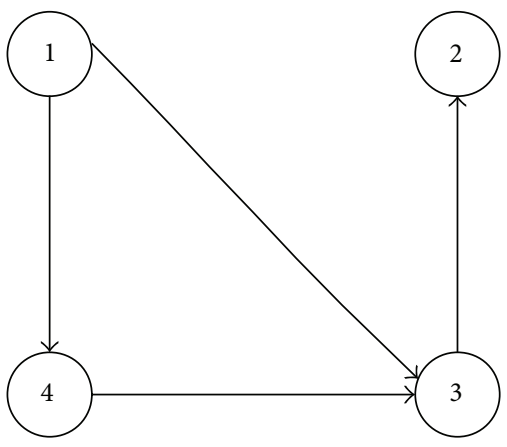

FIGURE 1: Topology.

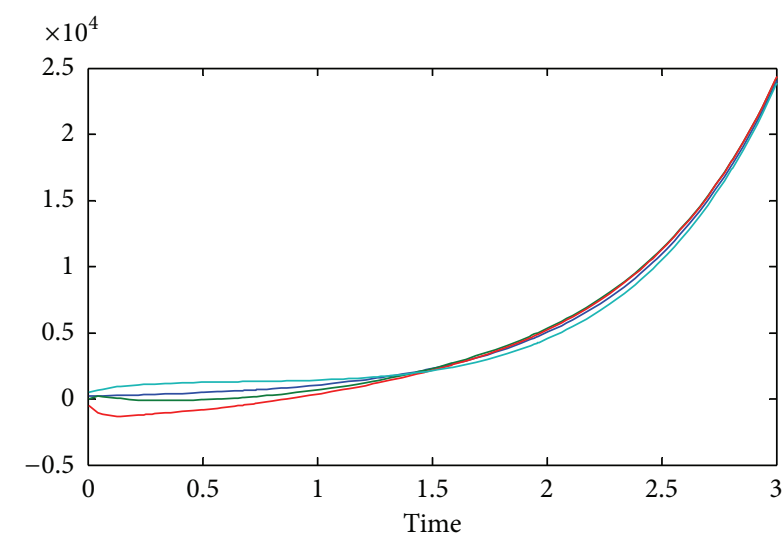

FIGURE 2: State trajectories $x_{11}, x_{21}, x_{31}, x_{41}$ during time interval $[0,3]$.

Theorem 4 shows that if $(A, B)$ is stabilizable and the topology graph has a spanning tree, then there exists controller gain $K$ and discrete times $t_{0}, t_{1}, \ldots$, where $t_{k+1}-t_{k} \leq d$, $k=0,1, \ldots$, such that consensus is reached. Moreover, $K$ and $d$ can be obtained by (11), which can be solved easily by the feasp solver in Matlab LMI Toolbox.

Similar to the analysis in the case of state feedback, the consensus under controller (4) is equivalent to the asymptotic stability of the following system:

$$
\dot{\dot{\delta}}(t)=\widehat{A} \hat{\delta}(t)+(H \otimes(B K C)) \zeta(t),
$$

where $\widehat{A}=I_{n-1} \otimes A-H \otimes(B K C), \zeta(t)=\widehat{\delta}(t)-\widehat{\delta}(t-\tau(t))$. By analyzing the stability of system (17), we obtain the following result.

Theorem 6. Assume that $(A, B, C)$ is stabilizable and detectable and the topology graph has a spanning tree. Controller (4) solves a consensus problem asymptotically if there exist positive definite matrices $P, R$ such that $K$ and $d$ satisfy the following LMI:

$$
\left(\begin{array}{ccc}
P \widehat{A}+\widehat{A}^{T} P & P(H \otimes(B K C)) & \widehat{A}^{T} R \\
* & -\frac{1}{d} R & (H \otimes(B K C))^{T} R \\
* & * & -\frac{1}{d} R
\end{array}\right) .
$$




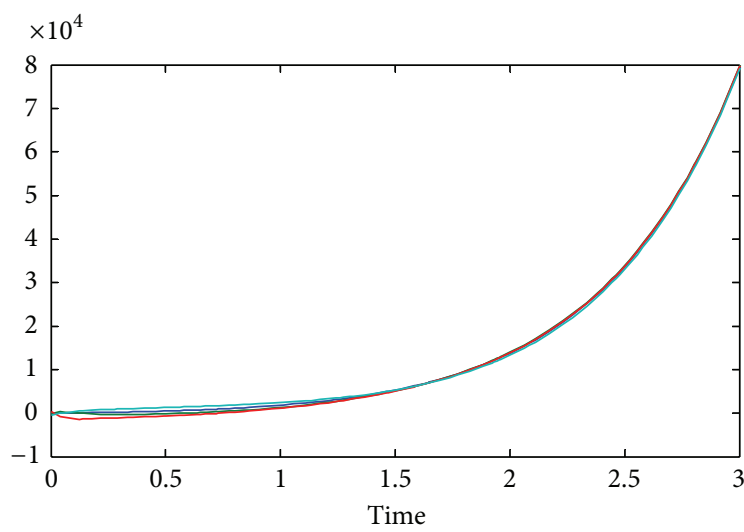

Figure 3: State trajectories $x_{12}, x_{22}, x_{32}, x_{42}$ during time interval $[0,3]$.

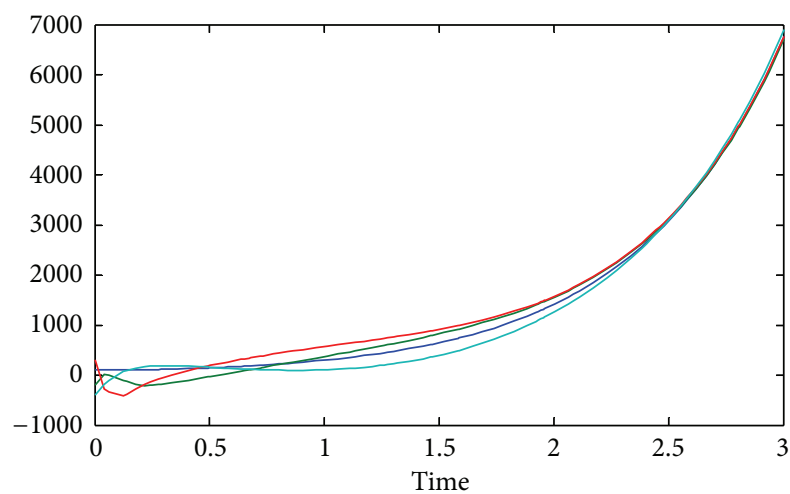

Figure 4: State trajectories $x_{13}, x_{23}, x_{33}, x_{43}$ during time interval $[0,3]$.

Remark 7. Although only the synchronous case is considered, the method in our paper can be applied to study the asynchronous case; namely, the discrete times of each agent are independent of the others.

\section{Simulations}

Consider the system of four agents, where the topology among four agents is shown in Figure 1. The dynamics of each agent are

$$
\dot{x}_{i}=A x_{i}+B u_{i}, \quad i=1,2,3,4,
$$

where $x_{i}=\left(x_{i 1}, x_{i 2}, x_{i 3}\right)^{T}$, and

$$
A=\left(\begin{array}{ccc}
1 & 0 & 2 \\
2 & 1 & 1 \\
1 & 0 & -2
\end{array}\right), \quad B=\left(\begin{array}{l}
1 \\
2 \\
1
\end{array}\right) .
$$

Since $\operatorname{rank}\left(B, A B, A^{2} B\right)=3,(A, B)$ is stabilizable. It is easy to verify that $\widetilde{A}$ is Hurwitz stable for $K=(-4,5,2)$. By using the feasp solver in Matlab LMI Toolbox, (11) is feasible for $d=$ 0.0625 and it is infeasible for $d=0.0626$, which means that for $K=(-4,5,2)$, the maximum $d$ satisfying (11) is $d=0.0625$.

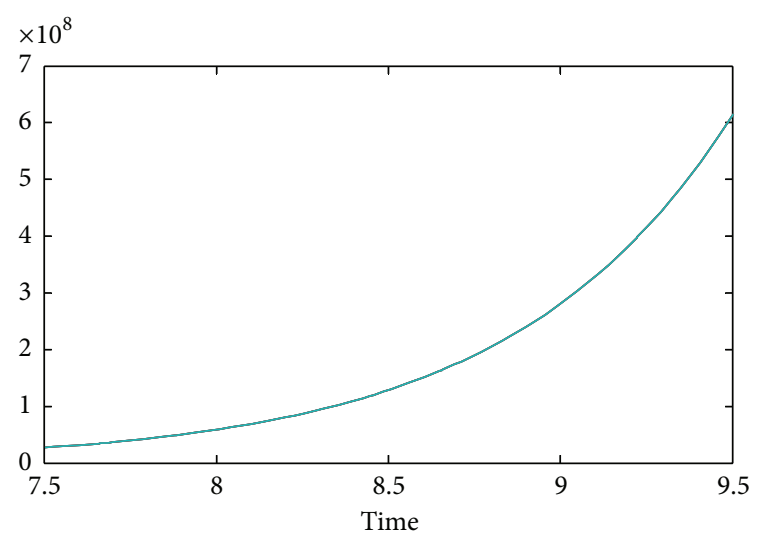

FIgURE 5: State trajectories $x_{11}, x_{21}, x_{31}, x_{41}$ during time interval $[7.5,9.5]$.

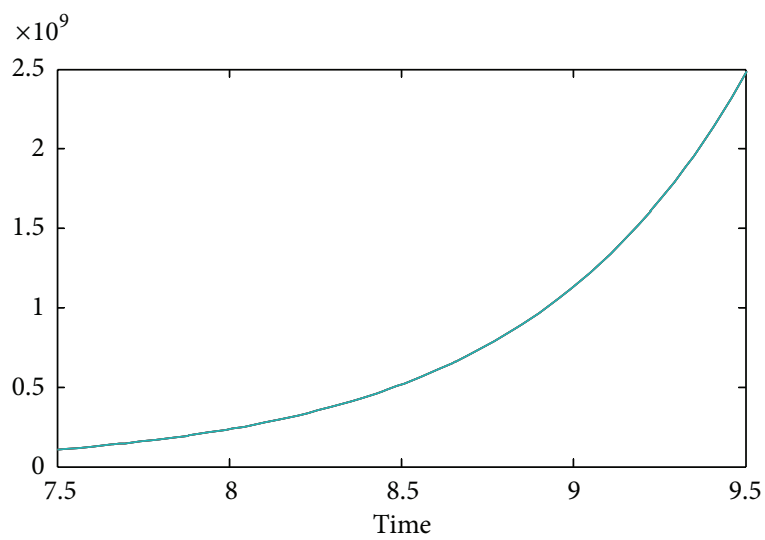

FIgURE 6: State trajectories $x_{12}, x_{22}, x_{32}, x_{42}$ during time interval $[7.5,9.5]$.

By Theorem 4 , for $K=(-4,5,2)$, consensus can be reached if the maximum discrete time interval is not larger than 0.0625 .

Without loss of generality, the discrete time intervals are chosen from $(0,0,0625]$ randomly. Then the state strategies of four agents during time interval $[0,3]$ are shown in Figures 2, 3 , and 4 , and the state strategies of four agents during time interval [7.5,9.5] are shown in Figures 5, 6, and 7, which validate our theoretical results.

\section{Conclusion}

This paper has studied the consensus problem of continuoustime multiagent systems with general linear dynamics and nonuniform sampling. By applying a state transformation and the input-delay approach, the consensus under consideration is equivalent to the asymptotic stability of a continuoustime system with time-varying delay. By analyzing the asymptotic stability of the continuous-time system, it is shown that there exist a controller gain and discrete times such that consensus can be reached. Furthermore, the controller gain and the upper bound of discrete time intervals can be obtained easily by solving a linear matrix inequality. 


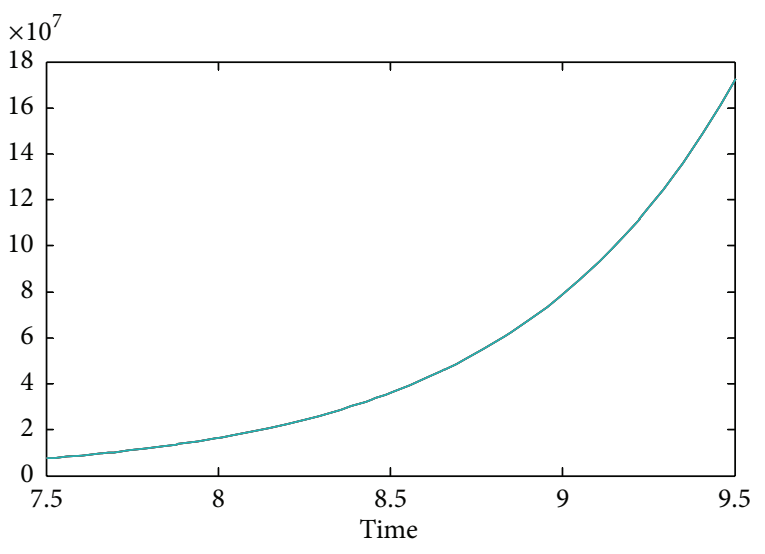

FIGURE 7: State trajectories $x_{13}, x_{23}, x_{33}, x_{43}$ during time interval $[7.5,9.5]$.

Simulations have been provided to illustrate the effectiveness of the theoretical results.

\section{Acknowledgments}

This work was supported by the National Natural Science Foundation (61170113, 61203150, and 61104141), Beijing Natural Science Foundation (4122019), the Science and Technology Development Plan Project of Beijing Education Commission (no. KM201310009011), and the Funding Project for Academic Human Resources Development in Institutions of Higher Learning under the Jurisdiction of Beijing Municipality (PHR201108055).

\section{References}

[1] R. Olfati-Saber and R. M. Murray, "Consensus problems in networks of agents with switching topology and time-delays," IEEE Transactions on Automatic Control, vol. 49, no. 9, pp. 1520$1533,2004$.

[2] W. Ren and R. W. Beard, "Consensus seeking in multiagent systems under dynamically changing interaction topologies," IEEE Transactions on Automatic Control, vol. 50, no. 5, pp. 655661, 2005.

[3] P. Frasca, "Continuous-time quantized consensus: convergence of Krasovskii solutions," Systems \& Control Letters, vol. 61, no. 2, pp. 273-278, 2012.

[4] L. Wang and F. Xiao, "Finite-time consensus problems for networks of dynamic agents," IEEE Transactions on Automatic Control, vol. 55, no. 4, pp. 950-955, 2010.

[5] Y. Zheng, W. Chen, and L. Wang, "Finite-time consensus for stochastic multi-agent systems," International Journal of Control, vol. 84, no. 10, pp. 1644-1652, 2011.

[6] N. Abaid and M. Porfiri, "Consensus over numerosityconstrained random networks," IEEE Transactions on Automatic Control, vol. 56, no. 3, pp. 649-654, 2011.

[7] F. Xiao and L. Wang, "Asynchronous consensus in continuoustime multi-agent systems with switching topology and timevarying delays," IEEE Transactions on Automatic Control, vol. 53, no. 8, pp. 1804-1816, 2008.
[8] P. Lin and Y. Jia, "Multi-agent consensus with diverse timedelays and jointly-connected topologies," Automatica, vol. 47, no. 4, pp. 848-856, 2011.

[9] P. Lin, K. Qin, H. Zhao, and M. Sun, "A new approach to average consensus problems with multiple time-delays and jointlyconnected topologies," Journal of the Franklin Institute, vol. 349, no. 1, pp. 293-304, 2012.

[10] Y. Sun, "Average consensus in networks of dynamic agents with uncertain topologies and time-varying delays," Journal of the Franklin Institute, vol. 349, no. 3, pp. 1061-1073, 2012.

[11] P. Lin and Y. Jia, "Consensus of a class of second-order multiagent systems with time-delay and jointly-connected topologies," IEEE Transactions on Automatic Control, vol. 55, no. 3, pp. 778-784, 2010.

[12] H. Liu, G. Xie, and L. Wang, "Necessary and sufficient conditions for solving consensus problems of double-integrator dynamics via sampled control," International Journal of Robust and Nonlinear Control, vol. 20, no. 15, pp. 1706-1722, 2010.

[13] Y. Cao and W. Ren, "Multi-vehicle coordination for doubleintegrator dynamics under fixed undirected/directed interaction in a sampled-data setting," International Journal of Robust and Nonlinear Control, vol. 20, no. 9, pp. 987-1000, 2010.

[14] Y. Gao and L. Wang, "Consensus of multiple dynamic agents with sampled information," IET Control Theory \& Applications, vol. 4, no. 6, pp. 945-956, 2010.

[15] A. Abdessameud and A. Tayebi, "On consensus algorithms for double-integrator dynamics without velocity measurements and with input constraints," Systems \& Control Letters, vol. 59, no. 12, pp. 812-821, 2010.

[16] P. Lin and Y. Jia, "Further results on decentralised coordination in networks of agents with second-order dynamics," IET Control Theory \& Applications, vol. 3, no. 7, pp. 957-970, 2009.

[17] F. Xiao, L. Wang, and J. Chen, "Partial state consensus for networks of second-order dynamic agents," Systems \& Control Letters, vol. 59, no. 12, pp. 775-781, 2010.

[18] D. Xie and S. Wang, "Consensus of second-order discrete-time multi-agent systems with fixed topology," Journal of Mathematical Analysis and Applications, vol. 387, no. 1, pp. 8-16, 2012.

[19] Y. Zhao, Z. Duan, G. Wen, and Y. Zhang, "Distributed finitetime tracking control for multi-agent systems: an observerbased approach," Systems \& Control Letters, vol. 62, no. 1, pp. 22-28, 2013.

[20] F. Jiang and L. Wang, "Consensus seeking of high-order dynamic multi-agent systems with fixed and switching topologies," International Journal of Control, vol. 83, no. 2, pp. 404420, 2010.

[21] P. Lin, Z. Li, Y. Jia, and M. Sun, "High-order multi-agent consensus with dynamically changing topologies and timedelays," IET Control Theory \& Applications, vol. 5, no. 8, pp. 976981, 2011.

[22] G. Wen, Z. Duan, W. Yu, and G. Chen, "Consensus of multiagent systems with nonlinear dynamics and sampled-data information: a delayed-input approach," International Journal of Robust and Nonlinear Control, vol. 23, no. 6, pp. 602-619, 2013.

[23] J. H. Seo, H. Shim, and J. Back, "Consensus of high-order linear systems using dynamic output feedback compensator: low gain approach," Automatica, vol. 45, no. 11, pp. 2659-2664, 2009.

[24] C.-Q. Ma and J.-F. Zhang, "Necessary and sufficient conditions for consensusability of linear multi-agent systems," IEEE Transactions on Automatic Control, vol. 55, no. 5, pp. 1263-1268, 2010. 
[25] L. Cheng, Z.-G. Hou, Y. Lin, M. Tan, and W. Zhang, "Solving a modified consensus problem of linear multi-agent systems," Automatica, vol. 47, no. 10, pp. 2218-2223, 2011.

[26] K. You and L. Xie, "Network topology and communication data rate for consensusability of discrete-time multi-agent systems," IEEE Transactions on Automatic Control, vol. 56, no. 10, pp. 2262-2275, 2011.

[27] Y. Zhao, Z. Duan, G. Wen, and G. Chen, "Distributed $H_{\infty}$ consensus of multi-agent systems: a performance region-based approach," International Journal of Control, vol. 85, no. 3, pp. 332-341, 2012.

[28] Z. Li, Z. Duan, and G. Chen, "Consensus of discrete-time linear multi-agent systems with observer-type protocols," Discrete and Continuous Dynamical Systems B, vol. 16, no. 2, pp. 489-505, 2011.

[29] F. Xiao and L. Wang, "Consensus problems for high-dimensional multi-agent systems," IET Control Theory and Applications, vol. 1, no. 3, pp. 830-837, 2007.

[30] T. Chen and B. Francis, Optimal Sampled-Data Control Systems, Springer, London, UK, 1996.

[31] W. P. M. H. Heemels, J. H. Sandee, and P. P. J. van den Bosch, "Analysis of event-driven controllers for linear systems," International Journal of Control, vol. 81, no. 4, pp. 571-590, 2008.

[32] C. Godsil and G. Royle, Algebraic Graph Theory, Springer, New York, NY, USA, 2001.

[33] X. Zhu, B. Chen, D. Yue, and Y. Wang, "An improved input delay approach to stabilization of fuzzy systems under variable sampling," IEEE Transactions on Fuzzy Systems, vol. 20, no. 2, pp. 330-341, 2012.

[34] Y. G. Sun, L. Wang, and G. Xie, "Average consensus in networks of dynamic agents with switching topologies and multiple timevarying delays," Systems \& Control Letters, vol. 57, no. 2, pp. 175183, 2008. 


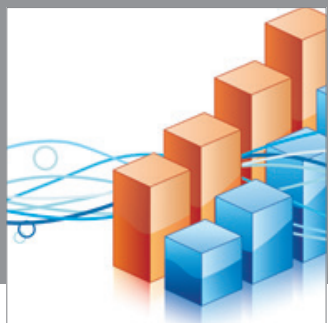

Advances in

Operations Research

mansans

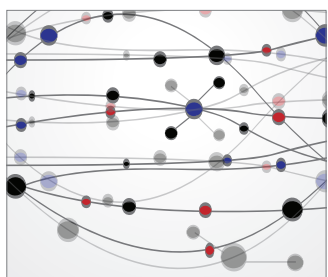

The Scientific World Journal
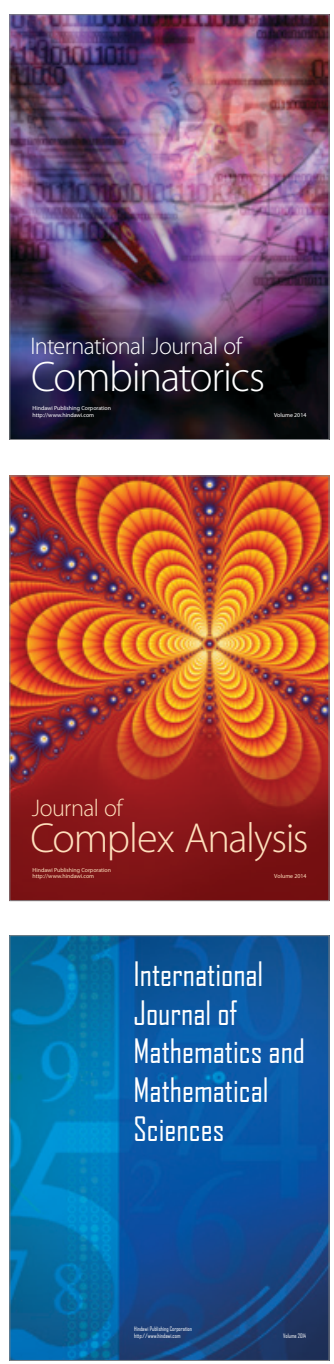
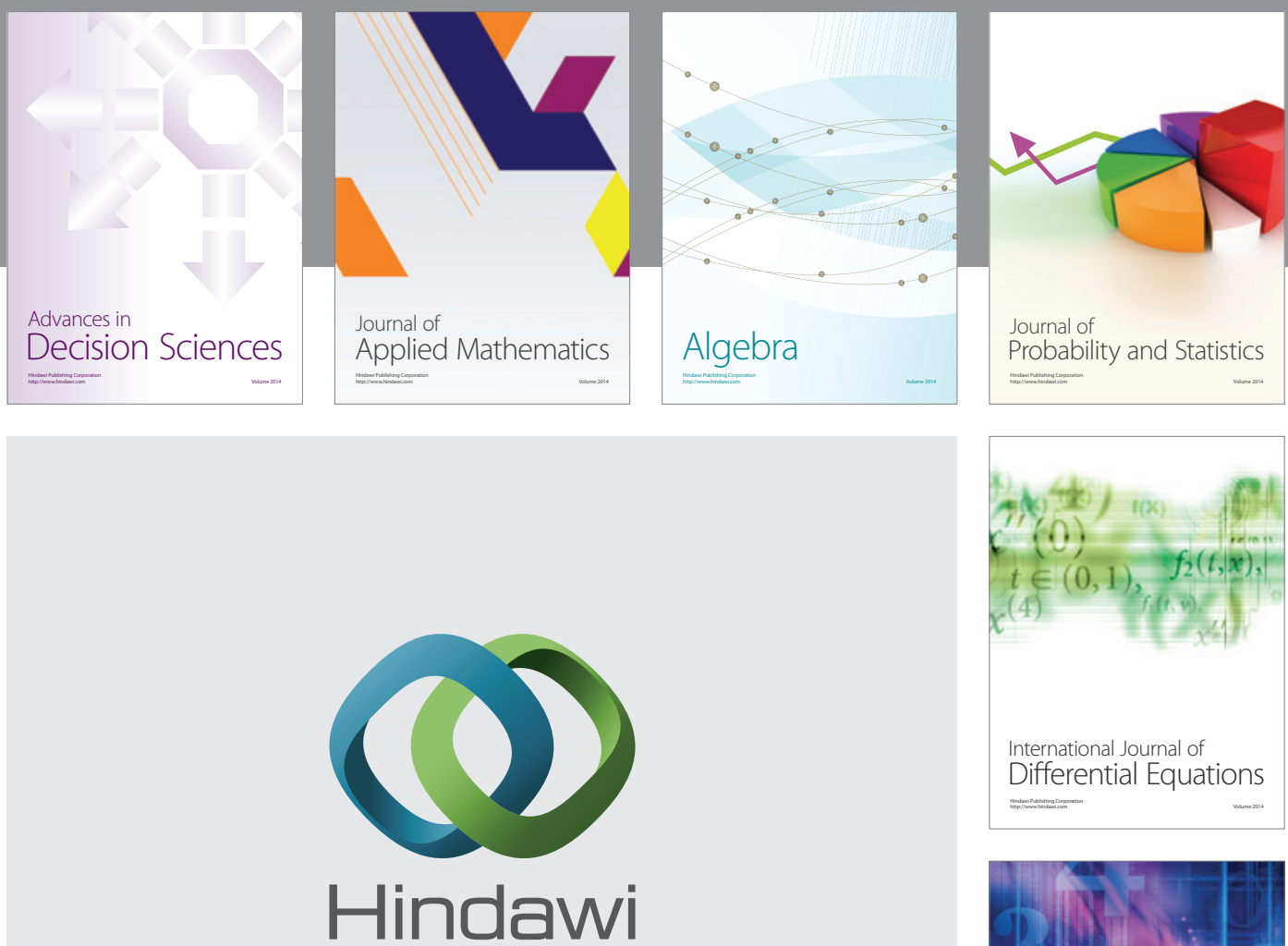

Submit your manuscripts at http://www.hindawi.com
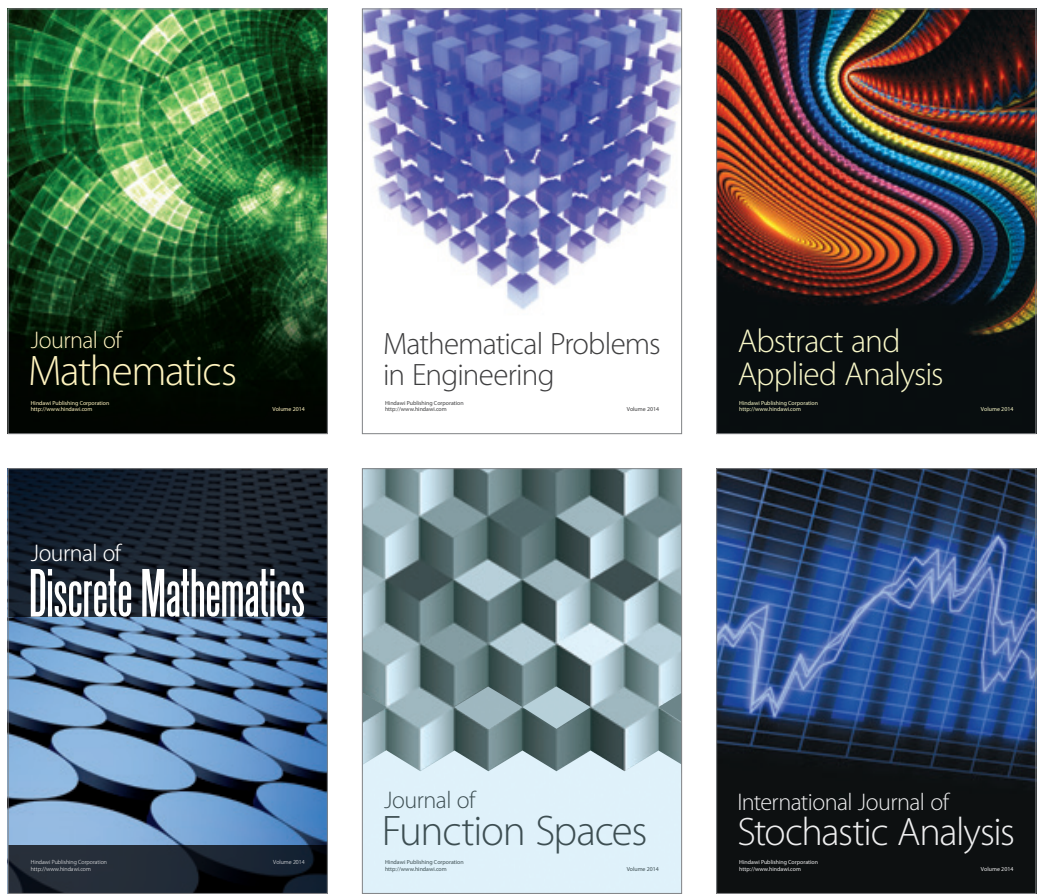

Journal of

Function Spaces

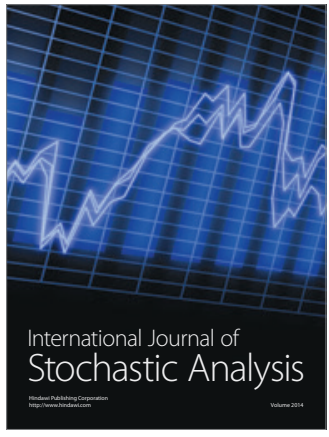

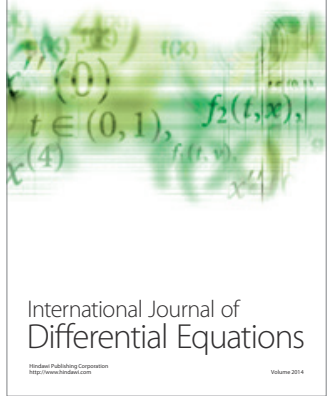
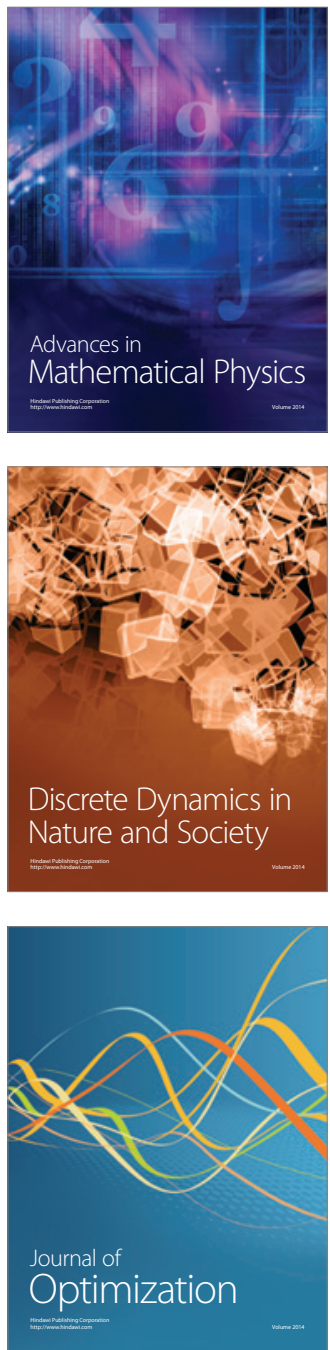